
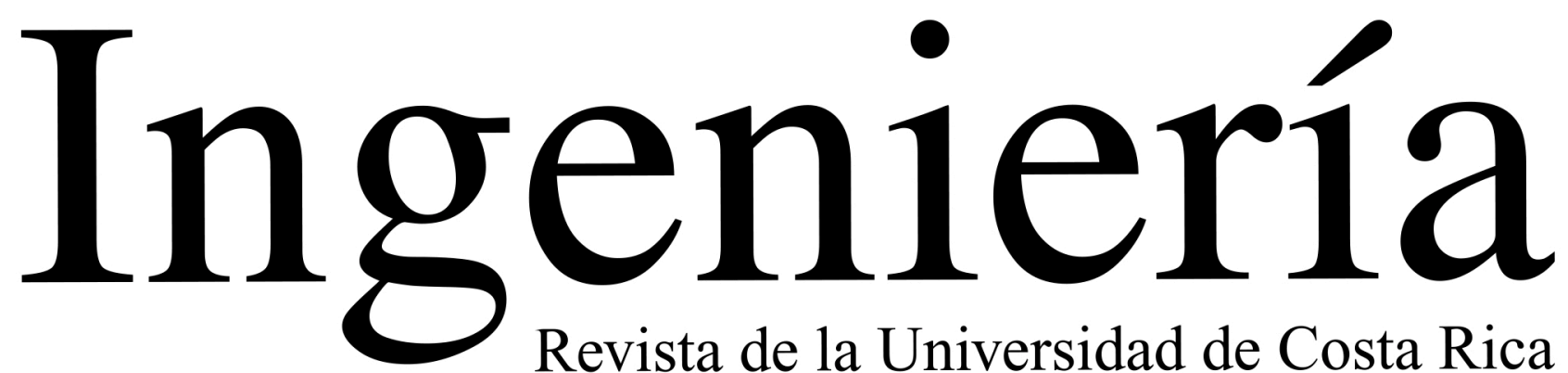

Revista de la Universidad de Costa Rica JULIO/DICIEMBRE 2021 - VOLUMEN 31 (2)

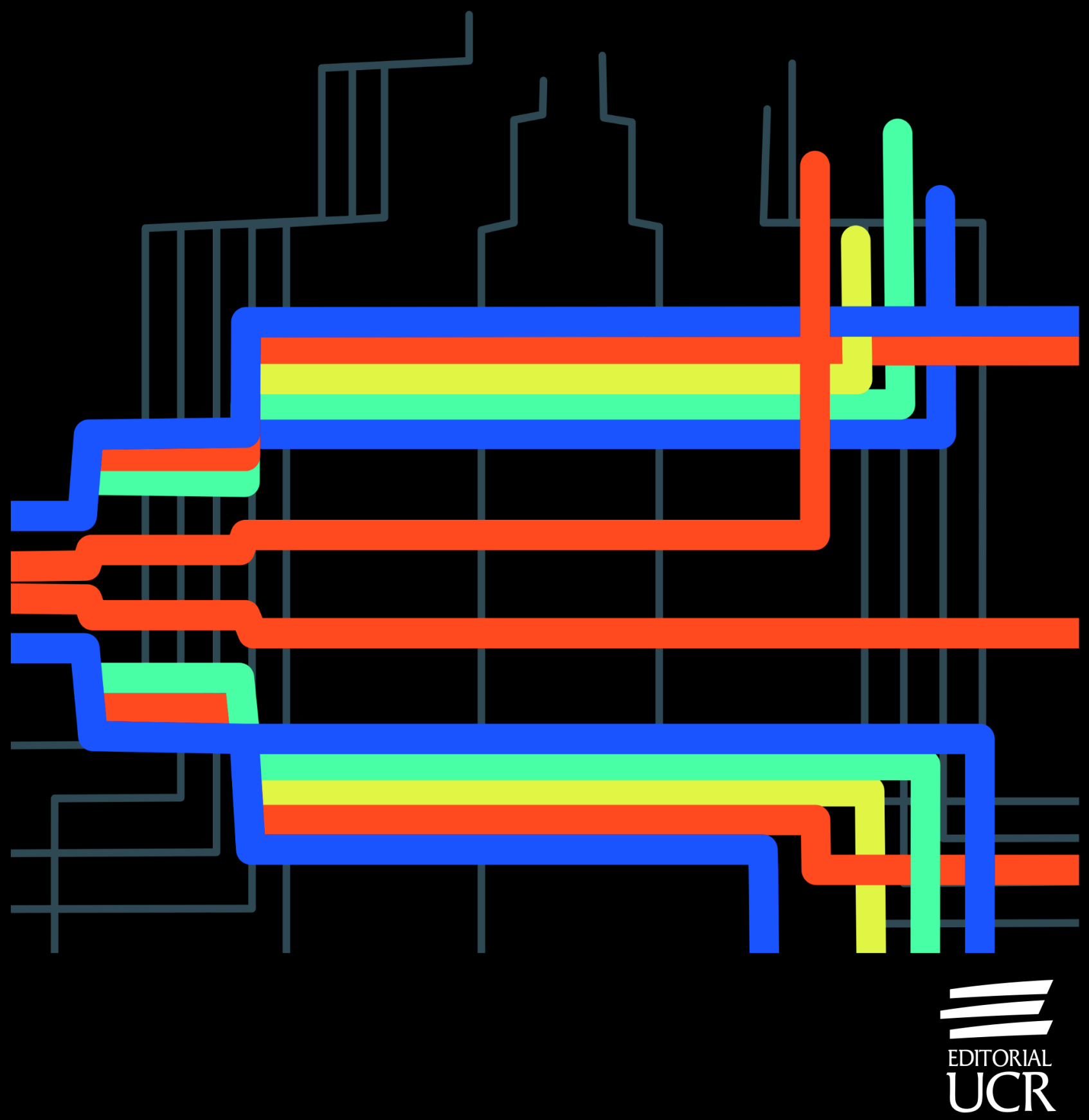




\title{
Análisis de la calidad de aguas de vertido en el Distrito de Riego Arenal-Tempisque (DRAT)
}

\author{
Wastewater quality analysis in Arenal-Tempisque Irrigation District \\ (DRAT)
}

\author{
Ing. Anayansi Wong Monge, \\ Escuela de Ingeniería de Biosistemas, \\ Universidad de Costa Rica, San José, Costa Rica \\ anayansi.wong@ucr.ac.cr \\ ORCID: 0000-0003-3539-310X \\ Ing. Alejandra María Rojas González, Ph.D., \\ Escuela de Ingeniería de Biosistemas, \\ Universidad de Costa Rica, San José, Costa Rica \\ alejandra.rojasgonzalez@ucr.ac.cr \\ ORCID: 0000-0001-7984-7789
}

\section{Resumen}

El Distrito de Riego Arenal-Tempisque (DRAT) es la dependencia gubernamental que regula la distribución del agua de riego en fincas agrícolas de la provincia de Guanacaste, Costa Rica. El DRAT monitorea semestralmente la calidad del agua de vertido utilizada en irrigación, generando reportes operacionales. Sin embargo, no es una práctica común realizar estudios donde se analice el comportamiento estadístico de los parámetros medidos en el tiempo para la toma de decisiones.

A partir de la caracterización de dos variables microbiológicas y veinte parámetros físico-químicos de la calidad del agua residual se realizó un análisis de estadística descriptiva para doce puntos de muestreo evaluados en un período de diez años (2008-2018). Se aplicó un análisis de componentes principales (ACP), donde los autovectores no mostraron diferencias apreciables para visualizar el peso de las variables de forma independiente. No obstante, el análisis permitió agrupar los parámetros más importantes que explican y describen el comportamiento del sistema estudiado como: la concentración de sales (Componente Principal 1 (CP1)); las sustancias activas al azul de metileno (SAAM) y el contenido de nitrógeno total (CP2); y la dinámica biológica (CP3); que se pueden relacionar con prácticas agrícolas. Adicionalmente, se llevó a cabo un análisis de conglomerados jerárquicos para los lugares en estudio y un análisis de discriminante, 
obteniéndose una caracterización de cuatro clúster, donde los resultados de ambas pruebas fueron concordantes con el ACP.

\title{
Palabras clave:
}

Análisis de componentes principales (ACP), análisis de conglomerados (AC), análisis de discriminante (AD), aguas de vertido, y sistemas de irrigación.

\begin{abstract}
The Arenal-Tempisque Irrigation District (DRAT by its acronym in Spanish) is the government agency that regulates the distribution of irrigation water in agricultural farms in the province of Guanacaste, Costa Rica. The DRAT have been monitoring the wastewater quality used by irrigation every six months, generating operational reports. However, it is not a common practice in irrigation districts to develop studies where parameters behavior had been analyzed over time.

A descriptive statistical analysis was carried out for 12 sampling points evaluated in ten years (20082018), based on the characterization of two microbiological and twenty physical-chemical variables of wastewater quality. A Principal Component Analysis (PCA) was applied, where eigenvector values did not show appreciable differences to visualize the weight of the variables independently. However, the analysis allowed grouping the most important parameters that explain and describe the studied system behavior, such as: salt concentration described by Principal Component 1 (CP1); methylene blue active substances (MBAS) and total nitrogen (CP2); and biological dynamics (CP3); that can be related to agricultural practices. Furthermore, a hierarchical cluster analysis was carried out for the study locations as well as a discriminant analysis, obtaining a characterization in four clusters, where the results of both tests agreed with the PCA.
\end{abstract}

\section{Keywords:}

Cluster analysis (CU), discriminant analysis (DA), irrigation systems, principal components analysis (PCA) and wastewaters. 


\section{INTRODUCCIÓN}

Alrededor del mundo, aproximadamente el $40 \%$ de la producción alimentaria procede de tierras agrícolas, de las cuales, cerca de un $20 \%$ es sometido a sistemas de riego. Los regadíos fomentan la productividad agrícola al permitir la intensificación de las cosechas durante el año y la diversificación de cultivos [1]. Los sistemas de irrigación tienen altos requerimientos del recurso hídrico, por lo que se debe tener un control de las características físico - químicas de las fuentes de agua [2].

La calidad del agua para el riego agrícola ha sido ampliamente investigada, pues ejerce un efecto directo en la producción de cultivos. Algunos de los problemas vinculados son: salinidad por concentración de sólidos disueltos, velocidad de infiltración del agua en el suelo que se asocia con la relación de adsorción de sodio (RAS), sólidos en suspensión que puede provocar que no sea apta para consumo humano o animal, contenido de nitratos y fosfatos, entre otros [3].

Altas concentraciones de sales generan restricciones abióticas que pueden impactar el balance del sistema suelo - agua [4]. Si se aplican láminas de riego y son zonas con evapotranspiración potencial (ETP) elevada, las sales se incrementan en el perfil del suelo. Consecuentemente, se provoca una reducción del agua disponible al aumentar el potencial osmótico de los suelos [5]. Los sólidos disueltos en el agua son medibles a través de la conductividad eléctrica.

Respecto a la salinidad de las aguas de irrigación y la relación de absorción de sodio (RAS), estas provocan un efecto interactivo con las propiedades físicas del suelo como: disminución en la conductividad hidráulica, dispersión de las arcillas, encostramiento superficial, entre otros [6]. La presencia de iones de calcio y de magnesio se relaciona con la dureza total del agua, generando incrustaciones o taponamientos en los sistemas cerrados de riego por aspersión. Es posible que provoque problemas de infiltración y precipitaciones en los suelos [7].

Los sistemas agrícolas de regadío son considerados generadores de contaminación difusa de aguas superficiales, pues pueden inducir problemas de eutroficación e hipoxia por excesos en concentración de sales de nitrógeno y fósforo por la aplicación de fertilizantes [8]

Según Pachepsky [9], la calidad microbiana de las aguas de irrigación puede ser afectada por diferentes fuentes: patrones climáticos [10], deyecciones animales [11], fuentes de contaminación difusa como la escorrentía que lava zonas de los agroecosistemas [12] o ingreso de aguas residuales sin tratamientos adecuados [13], prácticas agrícolas como abonos orgánicos y purines [14]. Además, se pueden producir cambios en la movilización del agua entre la fuente y los campos de cultivos. El deterioro microbiológico depende de la interacción del agua con reservorios de microorganismos en depósitos de sedimentos, bancos de suelos, algas y perifiton [15].

La estadística multivariada permite establecer las relaciones entre múltiples variables en sistemas complejos para simplificar y explicar su comportamiento. Entre algunas investigaciones relacionadas con la calidad del agua se menciona una evaluación de la dinámica de la calidad del agua con respecto a la variabilidad de los componentes espaciales y temporales, utilizando el análisis de clústers (AC), así como un análisis de componentes principales (ACP) y factoriales en Queensland, Australia [16]. En Egipto [17] se determinó un índice de calidad de agua de irrigación para regiones 
áridas y semiáridas usando análisis de correlación, el ACP y análisis factorial. Por otra parte, un estudio realizó un AC y un ACP para determinar la variabilidad espacial en la calidad del agua del lago Neusiedler See en Austria [18]; mientras que en India se evaluaron varias fuentes acuíferas para determinar si la calidad del agua era idónea para el consumo humano o bien para ser utilizada en irrigación, por medio de matrices de correlación y dendogramas [19]. A partir de análisis de conglomerados (AC) y análisis de discriminantes (AD) se evaluó la calidad del agua con respecto a variaciones espaciales y temporales en el sur de Florida [20]. Similarmente, en zonas estuarinas con presencia de manglares de Malasia se aplicaron modelos de regresión múltiple y AD [21].

En este estudio se plantea como objetivo realizar una evaluación estadística multivariada a partir de la caracterización de dos parámetros microbiológicos y veinte físico-químicos de los reportes operacionales semestrales de calidad de aguas de vertido para determinar las relaciones entre variables, su comportamiento y persistencia en el tiempo dentro del Distrito de Riego Arenal Tempisque (DRAT), Costa Rica.

\section{METODOLOGÍA}

\section{1 Área de Estudio}

El Distrito de Riego Arenal Tempisque (DRAT) se ubica en la provincia de Guanacaste en Costa Rica, cuyas coordenadas se muestran en la Fig. 1 y posee una altitud media de 63 m.s.n.m.

Al ubicarse dentro del Corredor Seco Centroamericano presenta un clima tropical seco. Además, según datos de la estación Hacienda Mojica del Instituto Meteorológico Nacional (IMN) [23], el régimen de precipitación predominantemente va de mayo a noviembre. Las mayores precipitaciones ocurren en octubre y setiembre, con valores de $265,5 \mathrm{~mm}$ y $244,1 \mathrm{~mm}$, respectivamente (Fig. 2). La estación Hacienda Mojica está localizada a $10^{\circ} 26^{\prime} 59^{\prime \prime}$ Norte y $85^{\circ} 10^{\prime}$ oeste, a una altitud de 33 m.s.n.m. Las temperaturas máximas se presentan entre los meses de febrero a mayo con máximas promedio de $34,8^{\circ} \mathrm{C}$ en abril, una temperatura media de $30,2^{\circ} \mathrm{C}$ y mínima de $25,5^{\circ} \mathrm{C}$ para el mismo mes.

En el complejo hidroeléctrico Arenal-Corobicí-Sandillal (ARCOSA) se localiza el Embalse Arenal, punto inicial de muestreo del DRAT. Las aguas son transportadas a tres casas de máquinas para la generación de energía, no son consideradas aguas residuales ordinarias, ni especiales; pues pasan por unidades turbogeneradoras sin provocar contaminación. Posteriormente, son conducidas hasta la presa derivadora Miguel Pablo Dengo Benavides (MPDB), donde se distribuyen a la red de canales. Esta red está conformada por el canal principal del sur y el canal del oeste, que se extienden por 8,5 y 21,9 km, respectivamente [24]. De ambos canales se despliegan derivaciones secundarias y terciarias, con una red de infraestructura hidroagrícola que incluye $255 \mathrm{~km}$ de canales de riego y $163 \mathrm{~km}$ de canales de drenaje como se presenta en la Fig. 1. 


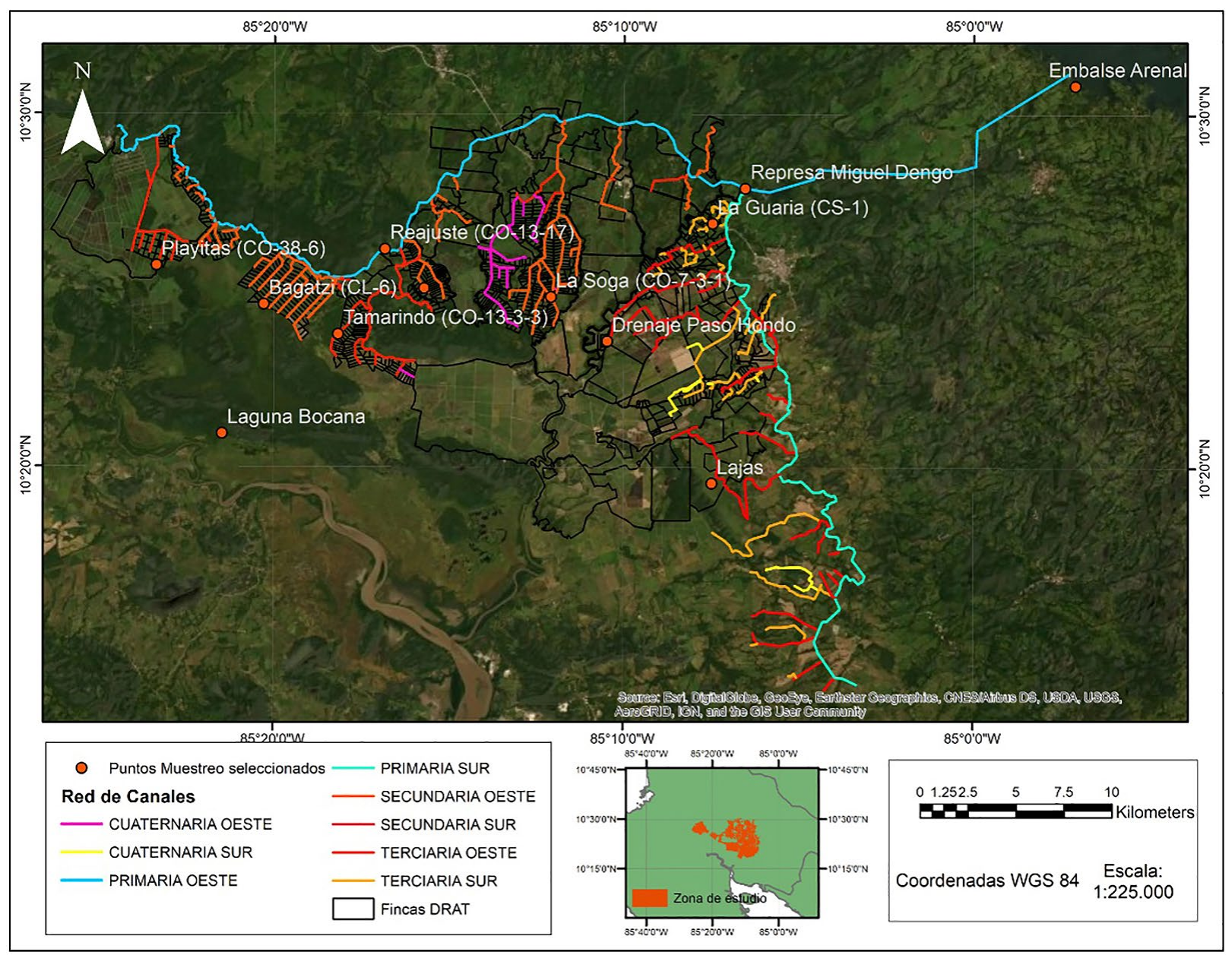

Fig. 1. Ubicación del DRAT, los puntos de muestreo evaluados y la distribución de la red de infraestructura hidroagrícola [22]

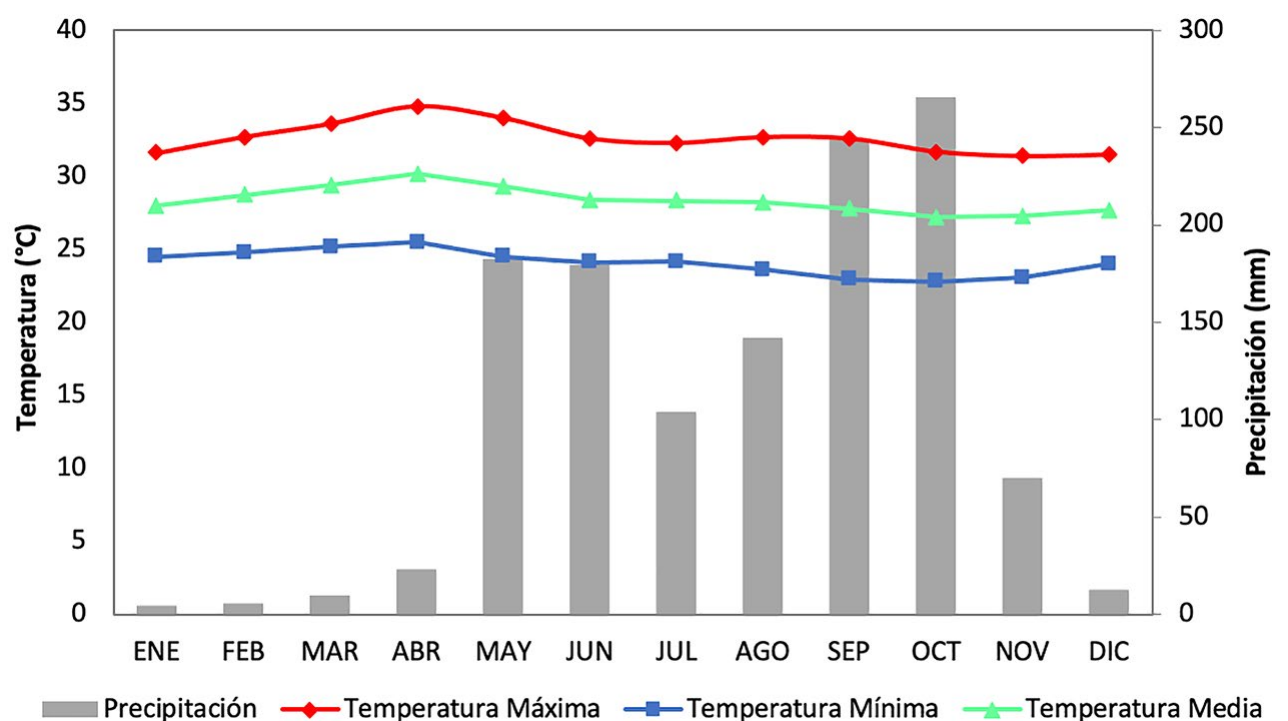

Fig. 2. Promedios mensuales del registro histórico (2000-2018) de la estación meteorológica Hacienda Mojica, en Bagaces, Costa Rica 


\subsection{Obtención de los datos y análisis estadístico}

El DRAT como un organismo regulador monitorea aguas superficiales, de pozos y vertido, realizando la caracterización físico-química de 29 puntos de control, de manera semestral. Las aguas de vertido son aquellas que han sido aprovechadas previamente en actividades agrícolas como el cultivo de arroz (13 687 ha), caña (11 888 ha), ganadería (2 641 ha), melón (1 800 ha) y cultivo de tilapia (571 ha), entre otras [25]. Los productores agrícolas son los responsables del uso y descarga de dichas aguas, que son evacuadas por procesos de escorrentía, infiltración de suelos a cuerpos receptores; sin aplicar ningún tipo de tratamiento.

El presente estudio se enfoca en el análisis de la información de calidad de agua en 12 ubicaciones que se aprecian en la Fig. 1. Para la evaluación se escogieron estos puntos de muestreo porque incluían datos de un período de diez años (2008-2018), lo cual hace representativa la muestra de datos. Los lugares que corresponden a sitios de vertido o descarga son: Bagatzí, Falconiana, La Guaria, La Soga, Lajas, Paso Hondo, Playitas, Reajuste (CO-13-17) y Tamarindo. Adicional, el DRAT complementa los reportes con análisis de aguas superficiales de los puntos de alimentación como lo son Embalse Arenal y presa derivadora MPDB; así como la Laguna Bocana que colinda con el DRAT, donde se realiza el monitoreo como un mecanismo de control para determinar que no se vean afectadas las aguas que se ubican en el Parque Nacional Palo Verde.

El Reglamento de Vertido y Reúso de Aguas Residuales (DE-33601MINAE-S) [26] de Costa Rica clasifica las aguas de vertido de los usuarios del DRAT de tipo especial por las actividades de ganadería (CIIU 01210) y piscicultura (CIIU 05002), que son vertidas en cuerpo receptor aplicando los límites máximos permisibles de las tablas 4 y 5 del decreto.

Las dos variables microbiológicas analizadas en este estudio corresponden a coliformes fecales (CF) y totales (CT) y las veinte variables físico-químicas analizadas son: demanda bioquímica de oxígeno (DBO), demanda química de oxígeno (DQO), nitrógeno total $(\mathrm{N})$, fósforo total $(\mathrm{P})$, dureza total (DT), calcio $\left(\mathrm{Ca}^{2+}\right)$, magnesio $\left(\mathrm{Mg}^{2+}\right)$, sodio $\left(\mathrm{Na}^{+}\right)$, potasio $\left(\mathrm{K}^{+}\right)$, sólidos disueltos $(\mathrm{SD})$, sólidos sedimentables (SS), sólidos suspendidos totales (SST), sólidos totales (ST), saturación de oxígeno (SO), sustancias activas al azul de metileno (SAAM), grasas y aceites (GyA). Además, la conductividad eléctrica (CE), el oxígeno disuelto (OD), el potencial de hidrógeno $(\mathrm{pH})$ y la temperatura (T), que fueron medidos en campo.

Para realizar los análisis se utilizaron los procedimientos del Standard Methods for the Examination of Water and Wastewater [27], a través del Laboratorio de Aguas del Centro de Investigación en Contaminación Ambiental (CICA) de la Universidad de Costa Rica. Se trabajó con una muestra de 22 datos para cada parámetro en evaluación para los diferentes puntos de muestreo.

Para la estadística descriptiva se calculó el promedio, la desviación estándar, la mediana y el máximo valor reportado. Para los coliformes fecales y totales se calculó el promedio geométrico y la desviación estándar geométrica, que se aplica en estudios de crecimiento bacteriológico, que presentan comportamientos logarítmicos. Los resultados fueron comparados con los valores máximos permitidos según el DE-33601-MINAE-S [26], que se muestran en la segunda columna de 
la Tabla 1. Algunos de los parámetros en estudio no se encuentran normados en Costa Rica, sin embargo, se cuantifican para un adecuado monitoreo del distrito de riego.

Para los análisis de estadística multivariada se emplearon los promedios de las variables originales, que fueron estandarizados para el análisis de componentes principales (ACP), se trabajó con una matriz de correlación y el estadístico KMO (Kaiser, Meyer y Olkin) [28].

Se manejó la totalidad de los datos para el análisis de conglomerados jerárquicos o clústers (AC), utilizando la distancia euclidiana para los puntos de muestreo, permitiendo la identificación del agrupamiento natural con base en las similitudes. La información obtenida fue empleada para la clasificación de los grupos para el análisis de discriminante (AD); aplicando el procedimiento estandarizado y la validación cruzada para estimar la tasa de error.

Para el tratamiento de los datos se manejaron tablas dinámicas de Excel, para el análisis estadístico se trabajó con Infostat [29] y el lenguaje de programación en R. Entre los programas utilizados se encuentran FactoMineR [30], ade4 [31] y mass [32].

\section{RESULTADOS Y DISCUSIÓN}

\subsection{Análisis de estadística descriptiva}

En la Tabla I se presentan los resultados de la estadística descriptiva aplicada a los análisis físicos, químicos y microbiológicos de la calidad de aguas de vertido en el DRAT, que permite evaluar la variabilidad, el comportamiento de los datos originales y el cumplimiento con la normativa nacional.

El análisis de coliformes fecales (CF) y totales (CT) que se muestran en la Tabla I, por ser variables microbiológicas se pueden trabajar con promedios geométricos. El punto de muestreo Embalse del Arenal y la represa MPDB presentan valores de 8 y 42 NMP/100 mL para CF, así como 27 y $180 \mathrm{NMP} / 100 \mathrm{~mL}$ para CT respectivamente, que son inferiores al límite del reglamento de 1 $000 \mathrm{NMP} / 100 \mathrm{~mL}$. Cabe destacar que ambos puntos son fuentes de agua del proyecto y obtienen valores menores con respecto a los otros puntos de medición. Los puntos que corresponden a tomas de drenaje que exceden el límite permitido de CF son: Bagatzí (1 500 NMP/ 100 mL) y Playitas (1 $269 \mathrm{NMP} / 100 \mathrm{~mL}$ ); entonces estas aguas vertidas presentan contaminación fecal.

Una evaluación de la composición química de las aguas se realiza por medio de la presencia de cationes. En el DRAT se evalúa: sodio $\left(\mathrm{Na}^{+}\right)$cuyas concentraciones varían de 1,35 a 5,00 mg/L; potasio $\left(\mathrm{K}^{+}\right)$de 4,62 a 55,47 mg/L; calcio $\left(\mathrm{Ca}^{2+}\right)$ de 10,41 a $32,38 \mathrm{mg} / \mathrm{L}$; y magnesio $\left(\mathrm{Mg}^{2+}\right)$ de 2,78 a 13,33 mg/L. La disolución de los dos últimos cationes se relaciona directamente con la dureza total que se reporta entre 33,93 a 80,79 mg/L (a excepción de la Bocana de 129,78 mg/L), por tanto al encontrarse por debajo de $120 \mathrm{mg} / \mathrm{L}$ se consideran aguas moderadamente duras según la clasificación de la Organización Mundial de la Salud [33].

En los puntos de evaluación, la conductividad eléctrica (CE) muestra un rango de variación entre 91,45 a 505,12 $\mu \mathrm{S} / \mathrm{cm}$ y los sólidos totales (ST) entre 89,98 a 456,85 mg/L. Ambos parámetros 
se relacionan con la concentración de sales presentes en el agua. Cabe mencionar que Heredia [5] recomienda determinar la cantidad y el origen de sales presentes para establecer los perjuicios potenciales que pueden ejercer en los cultivos y/o en los suelos.

La presencia de fósforo y nitrógeno total está normada en 25 y $50 \mathrm{mg} / \mathrm{L}$, respectivamente. Dichos parámetros se asocian con el proceso de eutroficación de los cuerpos de agua. Los promedios de los puntos de muestreo para fósforo total van de 0,04 a $0,42 \mathrm{mg} / \mathrm{L}$ y para nitrógeno total de 0,26 a 4,44 mg/L; o sea, que no exceden lo señalado en la ley. El punto de medición de Lajas reporta el promedio más alto de nitrógeno total, debido a un valor aislado muy alto donde se alcanzó el máximo de $50,60 \mathrm{mg} / \mathrm{L}$.

Los sólidos suspendidos totales (SST) consisten en coloides o sólidos de reducido tamaño de partícula $(<63 \mu \mathrm{m})$ que no sedimentan; provocando una limitación para la penetración de la luz, que ejerce influencia en la vida acuática [34]. En Costa Rica su máximo permisible es de $50 \mathrm{mg} / \mathrm{L}$ para la actividad agrícola. Los SST son excedidos por valores promedio en los siguientes puntos de muestreo: Bagatzí con un valor de 84,40 mg/L, La Bocana con 60,16 mg/L, Lajas con 64,90 $\mathrm{mg} / \mathrm{L}$, Playitas con 58,82 mg/L y Reajuste con 64,20 mg/L.

Si los sólidos suspendidos totales tienen una gravedad específica mayor de 1,0 se clasifican como sólidos sedimentables (SS), que según el reglamento [26] no debe sobrepasar el valor de 1 $\mathrm{mL} / \mathrm{L}$. Este parámetro es superado por los promedios en La Bocana (1,3 mL/L), Playitas (4,7 mL/L), Reajuste $(2,8 \mathrm{~mL} / \mathrm{L})$ y Tamarindo $(1,4 \mathrm{~mL} / \mathrm{L})$.

Los valores promedios de DBO -es decir, la cantidad de oxígeno que los organismos aerobios necesitan para procesar el material orgánico-se encuentran en un rango de 2,12 a 6,58 mg/L. Es decir, menos de la especificación máxima de $50 \mathrm{mg} / \mathrm{L}$. Asimismo, los promedios de DQO, que corresponden a la medición de oxígeno para oxidar la materia orgánica por agentes químicos, se mantienen entre 30,31 y 72,12 mg/L, por debajo del valor máximo de la regulación de $150 \mathrm{mg} / \mathrm{L}$.

La represa MPDB, fuente principal de agua al sistema, presenta un promedio \pm una desviación estándar para DBO de 2,47 $\pm 3,60 \mathrm{mg} / \mathrm{L}$ y para DQO de $41 \pm 44 \mathrm{mg} / \mathrm{L}$. Sin embargo, hay incumplimientos de DBO y DQO que se reflejan en los valores máximos reportados en Falconiana con 60 y $165 \mathrm{mg} / \mathrm{L}$ respectivamente; mientras que los siguientes puntos reportan valores máximos que sobrepasan solamente el parámetro de DQO: $273 \mathrm{mg} / \mathrm{L}$ para La Bocana, $194 \mathrm{mg} / \mathrm{L}$ para La Guaria, $212 \mathrm{mg} / \mathrm{L}$ para Paso Hondo y $231 \mathrm{mg} / \mathrm{L}$ para la represa MPBD. 


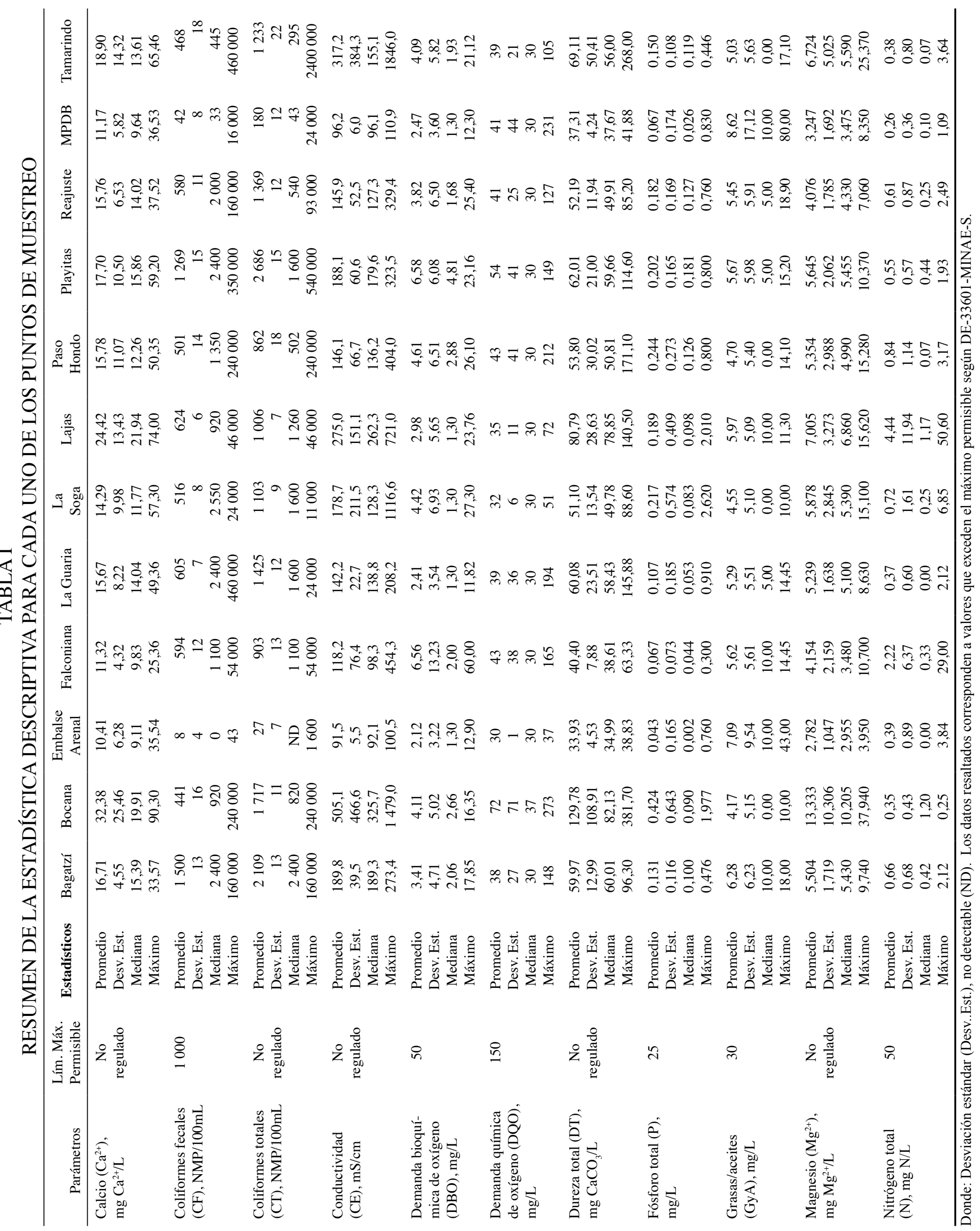




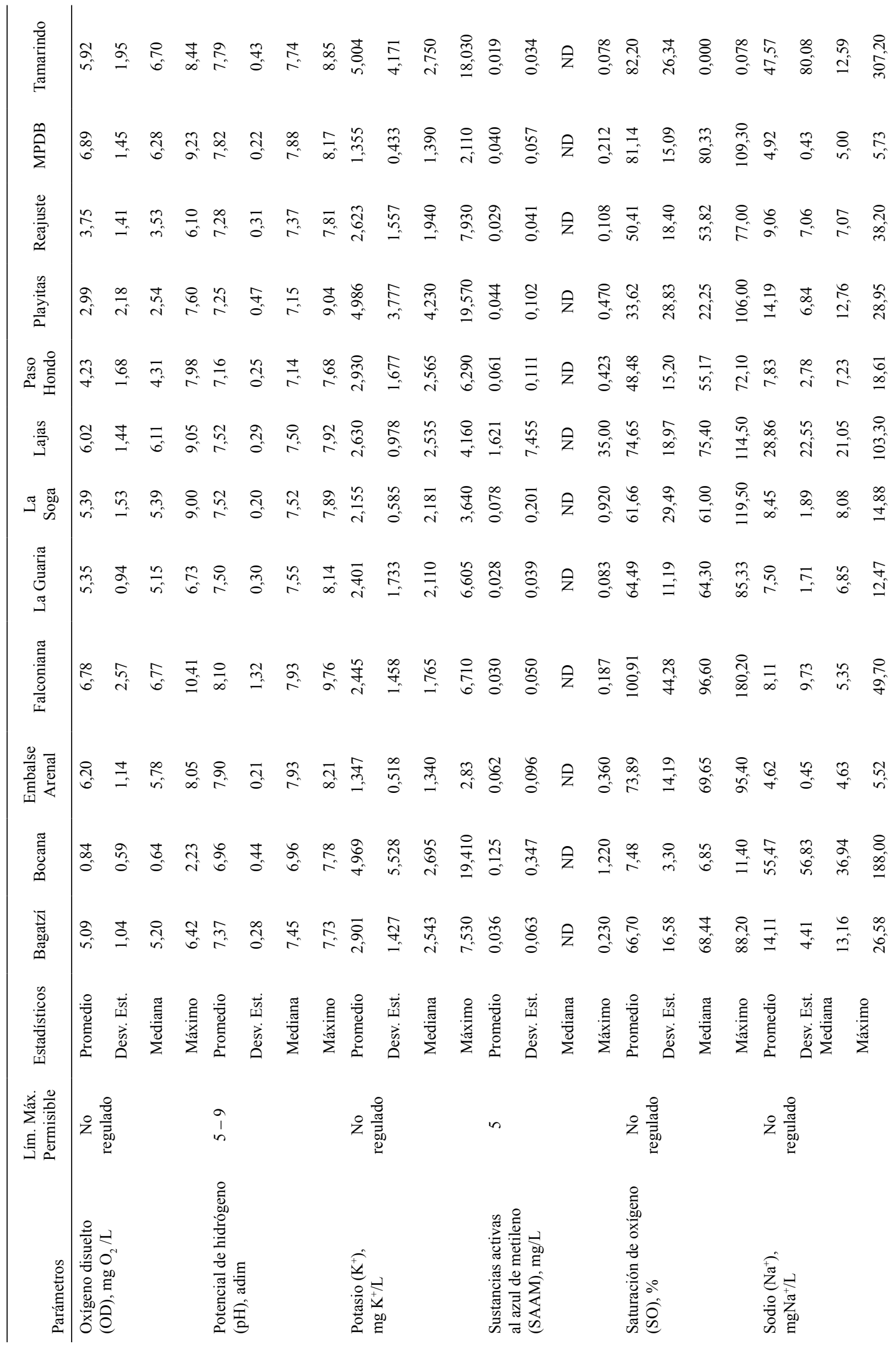




\begin{tabular}{|c|c|c|c|c|c|c|c|c|c|c|c|c|c|c|c|c|c|}
\hline 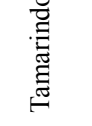 & $\begin{array}{l}\hat{\infty} \\
\vec{\lambda}\end{array}$ & $\stackrel{+}{+}$ & 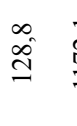 & $\overrightarrow{\underline{I}}=$ & $\stackrel{\Xi}{-}$ & के & $\therefore$ & $\begin{array}{ll}\dot{0} & \hat{0} \\
\hat{\sim} & \delta \\
\sigma\end{array}$ & है & $\begin{array}{l}\stackrel{2}{a} \\
\text { in }\end{array}$ & $\begin{array}{l}\stackrel{+}{+} \\
\stackrel{+}{d}\end{array}$ & $\begin{array}{l}\infty \\
\stackrel{\infty}{0} \\
\stackrel{0}{0}\end{array}$ & $\stackrel{+}{\stackrel{0}{0}}$ & 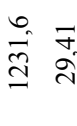 & $\stackrel{\infty}{-\infty}$ & \&. & $\begin{array}{l}8 \\
\dot{n} \\
m\end{array}$ \\
\hline 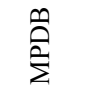 & $\stackrel{+}{i}$ & $\stackrel{\vec{d}}{\vec{d}}$ & $\vec{n}$ & $\stackrel{\circ}{\stackrel{0}{0}} \stackrel{0}{\stackrel{0}{0}}$ & $\therefore$ & $\because$ & $\therefore$ & $=\begin{array}{l}0 \\
0 \\
0\end{array}$ & $\stackrel{m}{n}$ & $\begin{array}{l}n \\
n \\
n\end{array}$ & $\begin{array}{l}\stackrel{8}{*} \\
\stackrel{2}{-}\end{array}$ & $\begin{array}{l}\infty \\
\infty \\
\infty\end{array}$ & $\stackrel{\infty}{\infty}_{\infty}^{\infty}$ & $\begin{array}{cc}0 & n \\
i & n \\
\Rightarrow & 0\end{array}$ & $\stackrel{\text { I }}{-}$ & $\begin{array}{l}\text { त् } \\
\text { d̦ }\end{array}$ & $\begin{array}{l}8 \\
\text { i } \\
\text { m }\end{array}$ \\
\hline 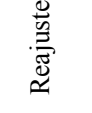 & $\overbrace{\beth}^{\text {తn }}$ & $\stackrel{\infty}{n}^{\circ}$ & $\begin{array}{l}\mathfrak{m}^{n} \\
=\end{array}$ & $\begin{array}{l}0 \\
\hat{d} \\
\text { id }\end{array}$ & $\stackrel{\infty}{i}$ & $\stackrel{\cong}{=}$ & $\therefore$ & 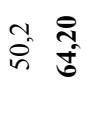 & $\stackrel{m}{\stackrel{m}{\infty}}$ & $\begin{array}{l}8 \\
\text { هi }\end{array}$ & $\begin{array}{l}8 \\
8 \\
0 \\
0\end{array}$ & to & $\stackrel{\sim}{\vec{i}}$ & 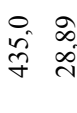 & 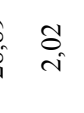 & $\begin{array}{l}8 \\
\stackrel{0}{0} \\
\infty\end{array}$ & $\begin{array}{l}8 \\
\text { n } \\
\text { m }\end{array}$ \\
\hline 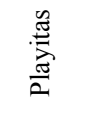 & $\begin{array}{l}n \\
\tilde{n} \\
n\end{array}$ & n. & त̂ & $\stackrel{+}{\stackrel{+}{+}}$ & f & $\stackrel{n}{\stackrel{1}{c}}$ & $\overrightarrow{0}$ & 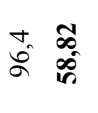 & $\begin{array}{l}\text { శ్ } \\
\text { ه్ర }\end{array}$ & 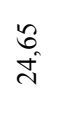 & 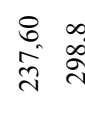 & $\begin{array}{l}\text { के } \\
\infty \\
\stackrel{\lambda}{\wedge}\end{array}$ & $\stackrel{+}{\stackrel{d}{d}}$ & 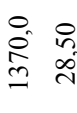 & $\stackrel{\infty}{\infty}$ & $\begin{array}{l}\stackrel{\infty}{\sim} \\
\stackrel{\infty}{\sim}\end{array}$ & $\begin{array}{l}\stackrel{\circ}{2} \\
\text { ले }\end{array}$ \\
\hline 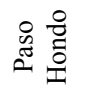 & $\begin{array}{l}\infty \\
\stackrel{0}{0}\end{array}$ & $\overrightarrow{+\infty}$ & $\begin{array}{l}\text { m } \\
\stackrel{0}{0}\end{array}$ & $\stackrel{\sim}{\stackrel{े}{d}}$ & $\stackrel{0}{\circ}$ & is & $\therefore$ & 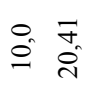 & $\begin{array}{l}\vec{b} \\
\text { i }\end{array}$ & $\begin{array}{l}\approx \\
\tilde{n}\end{array}$ & 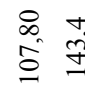 & $\begin{array}{l}\tilde{F} \\
\tilde{y}\end{array}$ & $\stackrel{+}{m}$ & 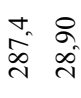 & $\stackrel{\infty}{\infty}$ & $\begin{array}{l}\text { ते } \\
\text { î } \\
\text { in }\end{array}$ & $\begin{array}{l}8 \\
\infty \\
\infty \\
m\end{array}$ \\
\hline 告 & $\hat{\sigma}$ & के & $\overrightarrow{\stackrel{i}{N}}$ & $\tilde{\beta}$ & $\begin{array}{l}0 \\
0 \\
0\end{array}$ & $\stackrel{+}{i}$ & $\overrightarrow{0}$ & $\stackrel{m}{=} \frac{8}{8}$ & $\begin{array}{l}\overrightarrow{\widehat{\widehat{S}}} \\
\text { }\end{array}$ & $\begin{array}{l}\text { ڤू } \\
\text { ¿े }\end{array}$ & 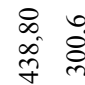 & $\stackrel{\infty}{\stackrel{\infty}{\Xi}}$ & 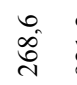 & 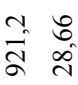 & $\stackrel{n}{n}$ & $\begin{array}{l}3 \\
0 \\
0 \\
0\end{array}$ & $\begin{array}{l}8 \\
\text { i }\end{array}$ \\
\hline تُ & 犬̃ & $\begin{array}{c}n \\
\approx \\
\infty\end{array}$ & $\begin{array}{l}\stackrel{0}{0} \\
\dot{s}\end{array}$ & $\begin{array}{l}0 \\
\dot{b} \\
\dot{b}\end{array}$ & $\overrightarrow{0}$ & Õ & $\overrightarrow{0}$ & 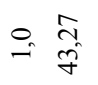 & $\begin{array}{l}\text { त̂ } \\
\text { s̊ }\end{array}$ & $\begin{array}{l}\stackrel{2}{0} \\
\stackrel{0}{0}\end{array}$ & $\begin{array}{l}\stackrel{\infty}{2} \\
\stackrel{2}{2}\end{array}$ & $\vec{a}$ & $\begin{array}{l}\infty \\
\stackrel{\Xi}{\simeq}\end{array}$ & 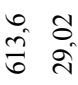 & 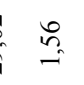 & $\begin{array}{l}\infty \\
0 \\
\infty \\
\infty\end{array}$ & $\begin{array}{l}8 \\
i \\
i\end{array}$ \\
\hline 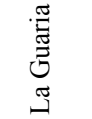 & ㅇ. & $\hat{\circ}$ & $\stackrel{\star}{\beth}$ & $\begin{array}{l}\infty \\
\infty \\
\infty \\
\infty\end{array}$ & $\therefore$ & $\therefore$ & $\therefore$ & $=\frac{a}{0}$ & $\begin{array}{l}\stackrel{2}{q} \\
\tilde{f}\end{array}$ & $\stackrel{R}{\stackrel{9}{0}}$ & $\begin{array}{l}\stackrel{0}{=} \\
\infty \\
\infty \\
\infty\end{array}$ & $\stackrel{3}{n}$ & $\begin{array}{l}+ \\
\stackrel{0}{0} \\
\stackrel{n}{n}\end{array}$ & 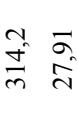 & $\stackrel{\infty}{\infty}$ & $\begin{array}{l}8 \\
\stackrel{0}{N}\end{array}$ & $\begin{array}{l}8 \\
\text { i }\end{array}$ \\
\hline 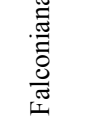 & $\stackrel{\forall}{\infty}$ & $\stackrel{0}{:}$ & $\stackrel{3}{\Omega}$ & $\stackrel{0}{\circ}$ & Oू. & $\stackrel{0}{\circ}$ & $\ddot{0}$ & $\begin{array}{ccc}0 & \vec{n} \\
\dot{m} & \tilde{n}\end{array}$ & $\begin{array}{l}\overrightarrow{\widehat{\theta}} \\
\vec{i}\end{array}$ & $\begin{array}{l}8 \\
\infty \\
\infty\end{array}$ & 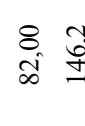 & $\stackrel{\vec{r}}{\stackrel{2}{i}}$ & $\begin{array}{l}\infty \\
\infty \\
\stackrel{0}{\unlhd}\end{array}$ & 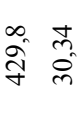 & $\frac{t}{i}$ & $\begin{array}{l}8 \\
\stackrel{0}{0}\end{array}$ & $\begin{array}{l}8 \\
\text { in } \\
\text { n. }\end{array}$ \\
\hline 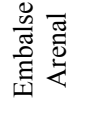 & के & $\hat{\sigma}$ & ले & $\stackrel{0}{\infty}$ & $\because$ & $\therefore$ & $\therefore$ & $=0$ तु & $\frac{m}{n}$ & 8 & $\stackrel{\text { ते }}{\hat{\sigma}}$ & m. & $\begin{array}{l}0 \\
\dot{\infty}\end{array}$ & 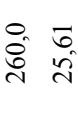 & $\stackrel{n}{=}$ & $\begin{array}{l}\text { g } \\
\text { a } \\
\text { in }\end{array}$ & $\begin{array}{l}8 \\
8 \\
8\end{array}$ \\
\hline 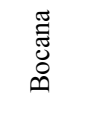 & $\begin{array}{l}0 \\
\stackrel{0}{0} \\
\text { in }\end{array}$ & $\hat{\tilde{n}}$ & 仓े & 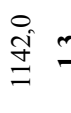 & 3 & $\vec{m}$ & $\overrightarrow{0}$ & $\begin{array}{l}0.0 \\
\stackrel{0}{8}\end{array}$ & $\begin{array}{l}\overrightarrow{0} \\
+ \\
+\end{array}$ & 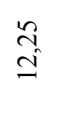 & $\begin{array}{ll}8 & \infty \\
\infty & 0 \\
\sim & \sigma\end{array}$ & $\underset{\substack{+\infty}}{\infty}$ & $\underset{\mathrm{i}}{\mathrm{i}}$ & 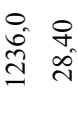 & $\stackrel{\infty}{\rightarrow}$ & $\begin{array}{l}\text { ते } \\
\text { ஸे }\end{array}$ & $\begin{array}{l}8 \\
i \\
i\end{array}$ \\
\hline 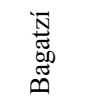 & $\begin{array}{l}0 \\
\infty \\
\stackrel{0}{0} \\
\end{array}$ & $\vec{g}$ & 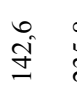 & $\begin{array}{l}\infty \\
\stackrel{n}{\sim}\end{array}$ & $\stackrel{\circ}{-}$ & $\stackrel{\circ}{i}$ & $\overrightarrow{0}$ & 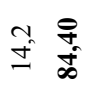 & 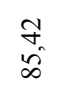 & $\begin{array}{l}\mathscr{\infty} \\
\infty \\
\tilde{\sigma}\end{array}$ & 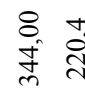 & \begin{tabular}{l}
0 \\
\multirow{a}{*}{}
\end{tabular} & $\begin{array}{l}\stackrel{\circ}{\infty} \\
\stackrel{\infty}{-}\end{array}$ & $\begin{array}{cc}0 & 0 \\
\infty & 0 \\
i n & 0\end{array}$ & $\stackrel{8}{8}$ & $\stackrel{\infty}{\infty}$ & $\begin{array}{l}8 \\
\dot{m} \\
m\end{array}$ \\
\hline 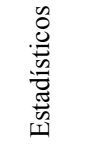 & 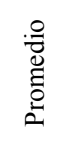 & 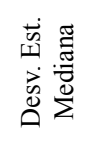 & 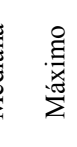 & & 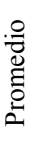 & 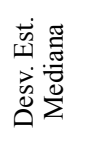 & 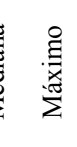 & 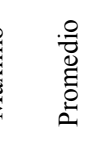 & 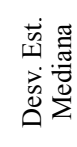 & 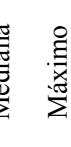 & 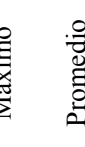 & 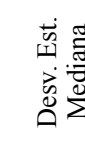 & 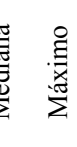 & 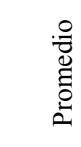 & 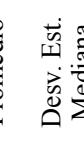 & & \\
\hline 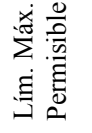 & $z$ & & & & - & & & in & & & & & & $\begin{array}{l}q \\
\stackrel{q}{\prime} \\
\end{array}$ & & & \\
\hline 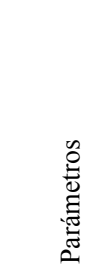 & 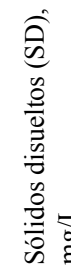 & & & & 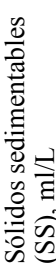 & & & 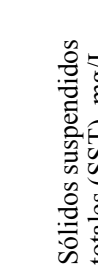 & & & 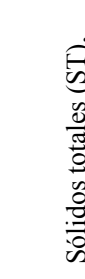 & & & 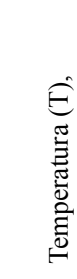 & 0 & & \\
\hline
\end{tabular}


Los ecosistemas acuáticos requieren de oxígeno disuelto en el agua para asegurar la vida de los organismos, la solubilidad del gas disminuye con aumentos de temperatura y de salinidad [35]. Según la Agencia de Protección Ambiental de los Estados Unidos (EPA) [36], la concentración mínima de oxígeno para mantener la biodiversidad de organismos acuáticos es de $5 \mathrm{mg} / \mathrm{L}$, valores menores de $3 \mathrm{mg} / \mathrm{L}$ son preocupantes y por debajo de $1 \mathrm{mg} / \mathrm{L}$ provocan una condición de hipoxia donde el ecosistema se caracteriza por la carencia de vida. En el DRAT se obtiene que Paso Hondo (4,23 $\mathrm{mg} / \mathrm{L})$, Playitas $(2,99 \mathrm{mg} / \mathrm{L})$ y Reajuste $(3,75 \mathrm{mg} / \mathrm{L})$ se encuentran en una situación comprometida para este parámetro; siendo la Bocana el sitio más crítico con una concentración de 0,84 mg/L.

La temperatura de los diferentes puntos de muestreo se mantuvo dentro del intervalo recomendado de 15 a $40{ }^{\circ} \mathrm{C}$, es un parámetro físico que se encuentra controlado.

Las concentraciones de grasas y aceites (GyA) y sustancias activas al azul de metileno (SAAM) no deben superar los $30 \mathrm{mg} / \mathrm{L}$ y $5 \mathrm{mg} / \mathrm{L}$ respectivamente [26]; son variables que se asocian con usos domésticos y/o industriales, que difieren de las prácticas agrícolas del DRAT, de tal modo que en la Tabla I no se observan incumplimientos en los canales de drenaje.

Cabe destacar que una de las limitaciones del estudio es que no se evaluaron cambios de las variables por estacionalidad, siendo un parámetro deseable a considerar. En algunos sitios de muestreo no se realizaron determinaciones en la época seca por no presentar caudal suficiente de vertido para la determinación.

Respecto a Embalse del Arenal, este presenta la mejor calidad de agua microbiológica, física y química; así, todos sus parámetros se encuentran dentro del rango de las especificaciones legales del país. Este punto de muestreo, junto con la represa MPDB, revela los menores índices de concentración de contaminantes. Ambos sitios reflejan que las fuentes de alimentación de agua del DRAT tienen un comportamiento diferenciado del resto de puntos (tomas de drenaje de aguas).

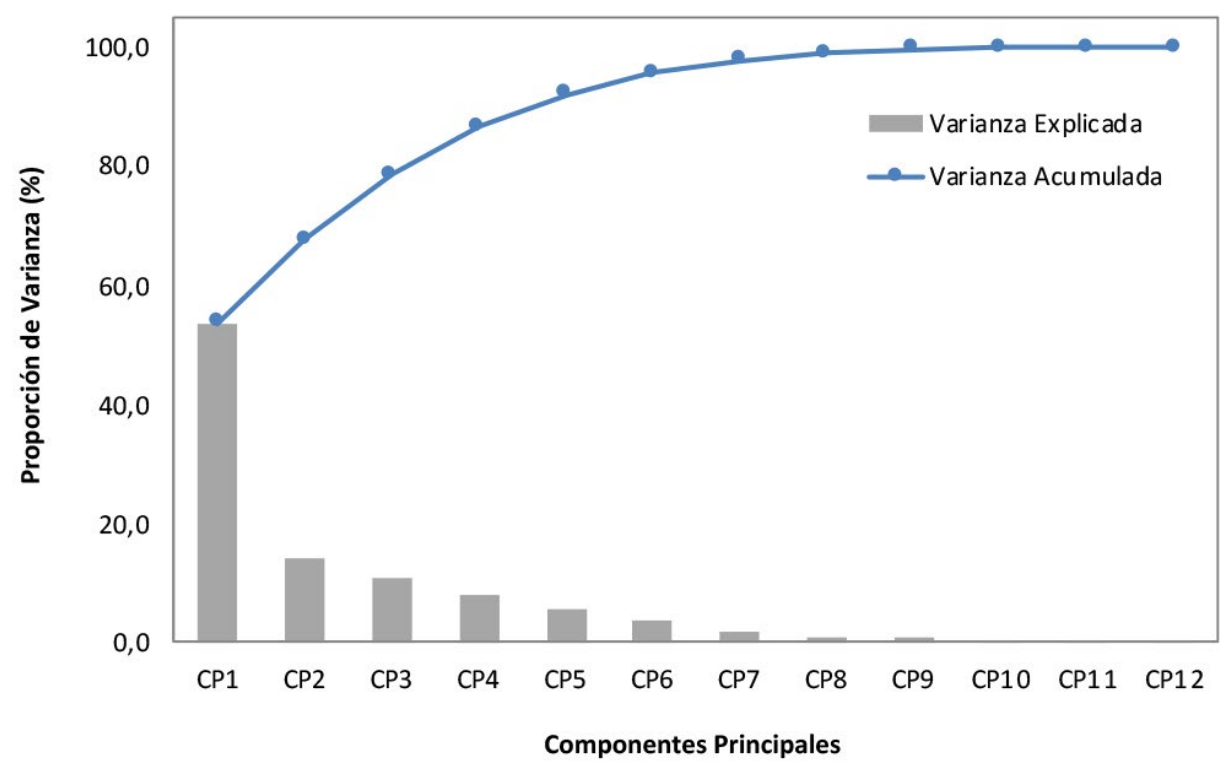

Fig. 3. Gráfico de la varianza explicada y acumulada por los componentes principales 


\subsection{Análisis de componentes principales (ACP)}

Las variables originales son estandarizadas, se calcula una matriz de correlación (Tabla II); donde se aprecian elementos que se encuentran fuertemente asociados entre sí, con base en el criterio de correlación de Pearson $(r>0,70)$ [37]. Estos resultados son pertinentes para la aplicación del análisis de componentes principales.

En la Fig. 3 se muestra la varianza asociada con el primer componente que explica el 53,68\%, el segundo el 13,98\% y el tercero el 11,03\%. En conjunto describen el 78,68\% del comportamiento de la calidad del agua de vertido del DRAT. Por tanto, se decide trabajar con estos tres componentes, pues la varianza acumulada es cercana al estándar del 80\% [37]. Para los tres primeros componentes principales se obtienen autovalores de 11,$81 ; 3,07$ y 2,43 respectivamente; todos por encima del promedio del valor de uno de la matriz de correlación.

En la Tabla III se exponen los resultados de los autovectores normalizados y se aprecia que las variables con los coeficientes más altos para el primer componente principal (CP1) corresponden a sólidos totales, sólidos disueltos, dureza total, magnesio, calcio y conductividad, que pueden ser interpretados como la concentración de sales o sólidos disueltos presentes en las aguas.

Los gráficos de dos dimensiones para los componentes principales y sus respectivos comportamientos individuales se presentan en la Fig. 4. El CP1 está definido en el eje horizontal de la Fig. 4.a y se puede observar el agrupamiento de las variables que más se correlacionan hacia la derecha (color verde) y cómo impacta en la mayoría de los puntos de muestreo (Fig. 4.b). El comportamiento descrito se reafirma en el gráfico 4.c. y 4.d.

Para el segundo componente principal (CP2) se observa en la Fig. 4.a que está altamente relacionado con las sustancias activas al azul de metileno (SAAM) y la concentración de nitrógeno total (N) que se ubican en el eje vertical en la dirección positiva. En el gráfico 4.b y 4.f se aprecia como el punto de evaluación Lajas se correlaciona fuertemente con los análisis de SAAM; que determina la presencia de sustancias jabonosas. Esto podría deberse a que algunos canales previos son alimentados con aguas provenientes de los ríos Higuerón y Cañas, en cuyos márgenes se ubican poblaciones.

En el tercer componente principal (CP3) se agrupan la temperatura (T), la demanda bioquímica de oxígeno (DBO), coliformes totales (CT) y la saturación de oxígeno (SO). En conjunto se pueden relacionar con la dinámica biológica, estas variables se vinculan con la presencia de oxígeno en las aguas necesario para el desarrollo de la vida acuática, incluyendo la presencia de microorganismos que consumen oxígeno para oxidar el material orgánico. El CP3 se ubica en las Fig. 4.c y 4.e en el eje de las "y", donde las variables que más se correlacionan con este componente, se ubican en ambos casos en la parte superior (color azul).

Para el CP3 y los gráficos de coordenadas individuales se identifica un impacto en la Laguna Bocana (Fig. 4.b y 4.d), que se ubica en el Parque Nacional Palo Verde, el cual colinda con tierras beneficiadas por el DRAT. Este humedal muestra una clara estacionalidad, ya que en la época seca se pueden observar ecosistemas acuáticos que se reducen y algunos pueden desaparecer [38]. 


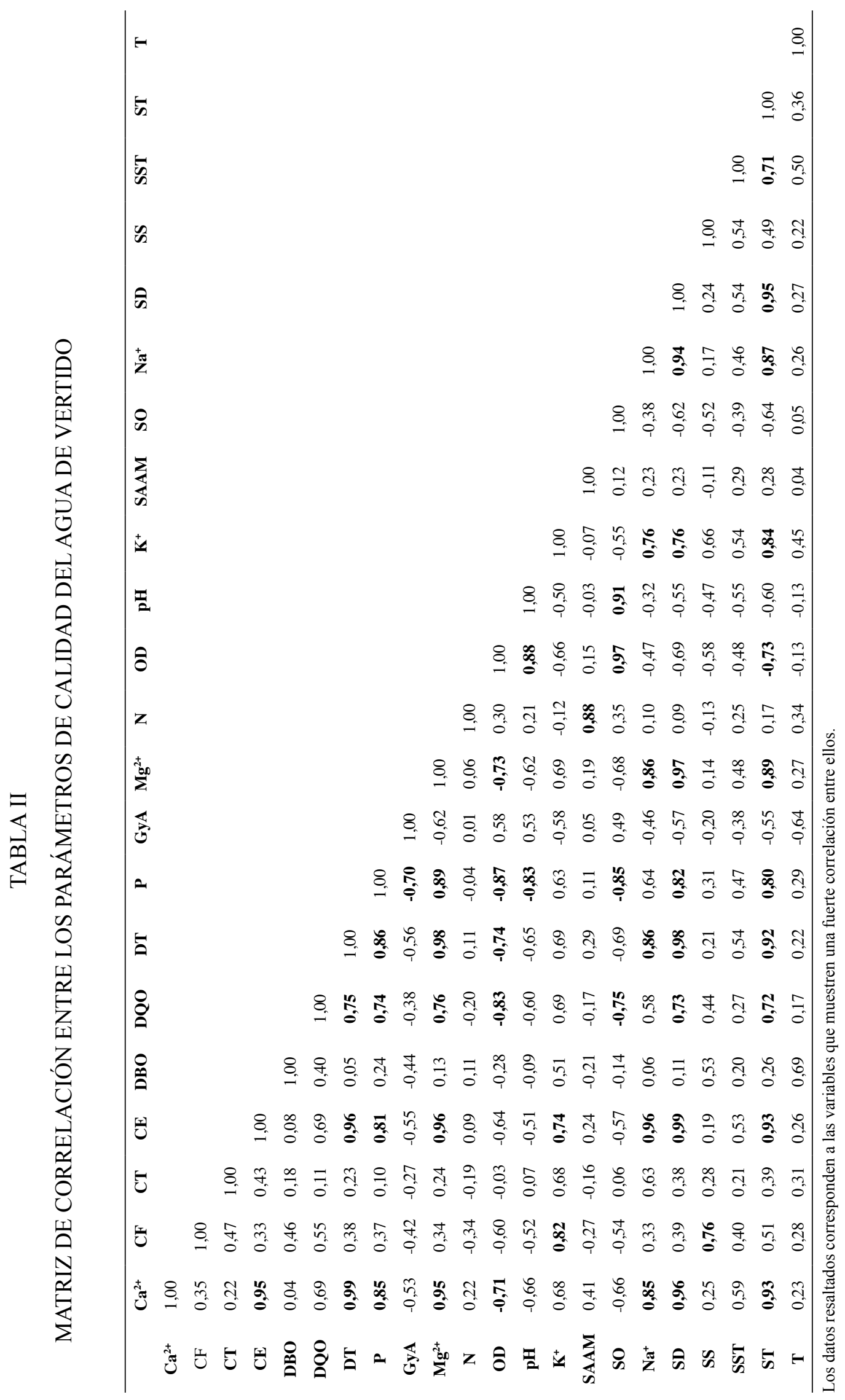


TABLA III

MATRIZ DE COMPONENTES PRINCIPALES CON LOSVALORES DE LOS AUTOVECTORES NORMALIZADOS

\begin{tabular}{cccc}
\hline Parámetros & $\mathbf{C P 1}$ & $\mathbf{C P 2}$ & $\mathbf{C P 3}$ \\
\hline Calcio $\left(\mathrm{Ca}^{2+}\right)$ & $\mathbf{- 0 , 2 7}$ & 0,20 & 0,08 \\
Coliformes fecales (CF) & $-0,18$ & $-0,34$ & $-0,15$ \\
Coliformes totales (CT) & $-0,10$ & $-0,06$ & $\mathbf{- 0 , 3 5}$ \\
Conductividad eléctrica (CE) & $\mathbf{- 0 , 2 7}$ & 0,18 & 0,02 \\
Demanda bioquímica de oxígeno (DBO) & $-0,09$ & $-0,25$ & $\mathbf{- 0 , 4 0}$ \\
Demanda química de oxígeno (DQO) & $-0,23$ & $-0,13$ & 0,12 \\
Dureza total (DT) & $\mathbf{- 0 , 2 7}$ & 0,16 & 0,10 \\
Fósforo total (F) & $-0,26$ & 0,01 & 0,15 \\
Grasas y aceites (GyA) & 0,20 & 0,06 & 0,14 \\
Magnesio (Mg ${ }^{2+}$ ) & $\mathbf{- 0 , 2 7}$ & 0,14 & 0,09 \\
Nitrógeno total (N) & 0,01 & $\mathbf{0 , 4 3}$ & $-0,26$ \\
Oxígeno disuelto (OD) & 0,25 & 0,19 & $-0,20$ \\
Potencial de hidrógeno (pH) & 0,22 & 0,13 & $-0,24$ \\
Potasio (K') & $-0,25$ & $-0,14$ & $-0,22$ \\
Sustancias activas al azul de metileno (SAAM) & $-0,03$ & $\mathbf{0 , 4 7}$ & $-0,07$ \\
Saturación de oxígeno (SO) & 0,23 & 0,18 & $\mathbf{- 0 , 3 1}$ \\
Sodio (Na ${ }^{+}$) & $-0,24$ & 0,19 & $-0,08$ \\
Sólidos disueltos (SD) & $\mathbf{- 0 , 2 7}$ & 0,15 & 0,03 \\
Sólidos sedimentables (SS) & $-0,14$ & $-0,31$ & $-0,17$ \\
Sólidos suspendidos totales (SST) & $-0,19$ & 0,06 & $-0,18$ \\
Sólidos totales (ST) & $\mathbf{- 0 , 2 8}$ & 0,10 & $-0,07$ \\
Temperatura (T) & $-0,11$ & $-0,01$ & $\mathbf{- 0 , 4 8}$ \\
\hline
\end{tabular}

Los datos resaltados corresponden a los parámetros incluidos en los componentes principales.

El ACP llevado a cabo demuestra que algunas variables están estrechamente relacionadas entre ellas como: la dureza total que encierra los iones de calcio y de magnesio; la determinación de oxígeno disuelto y la saturación de oxígeno; los sólidos disueltos y totales con la conductividad eléctrica. De tal forma que podrían eliminarse algunas de las mencionadas porque brindan información redundante y no son parámetros obligatorios según DE33601-MINAE-S. 


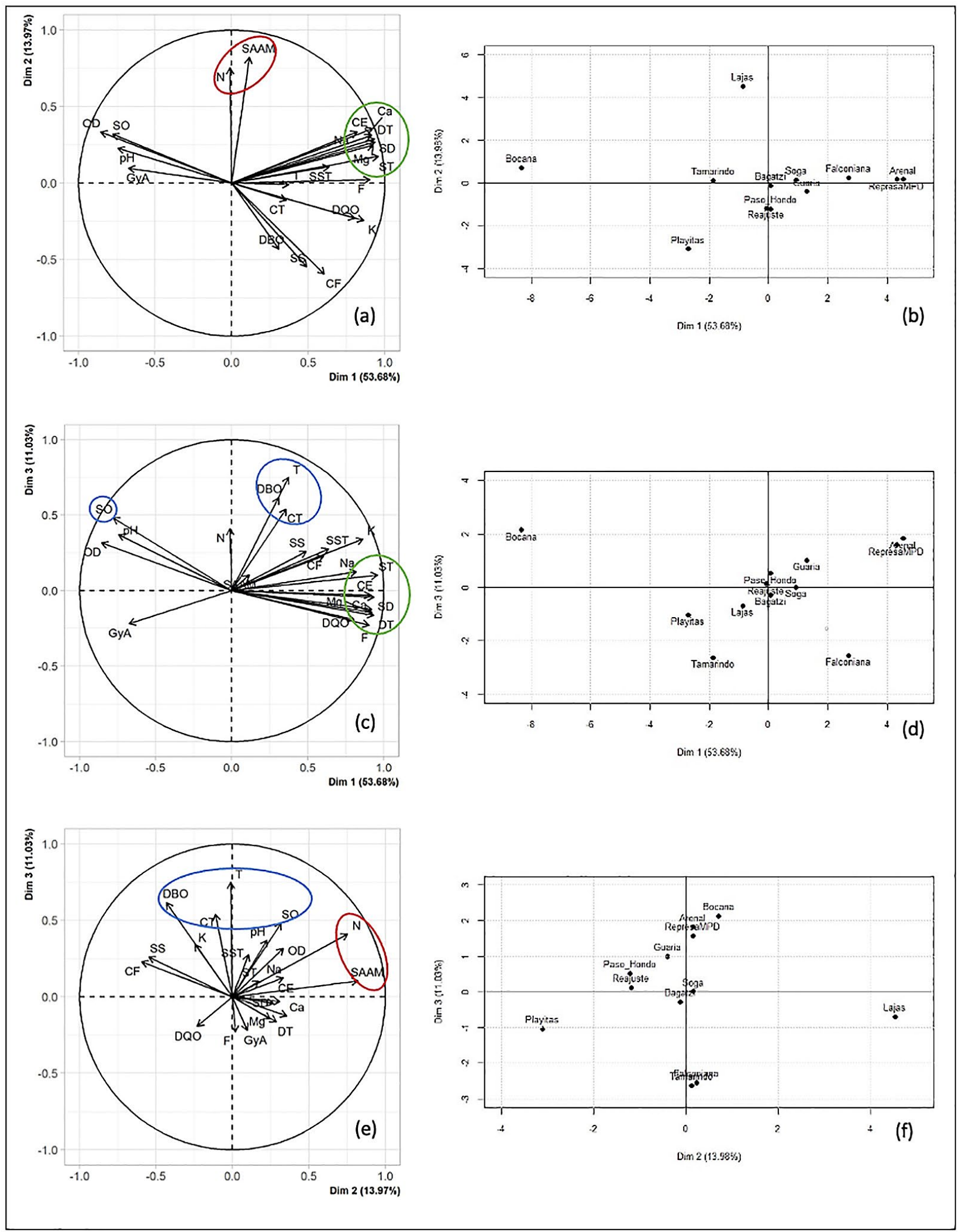

Fig. 4. Gráficos en dos dimensiones para los componentes principales CP1 y CP2 (a), CP1 y CP3 (c), CP2 y CP3 (e) y las coordenadas de los lugares en el plano respectivas a cada par evaluado se ubican en (b), (d) y (f), respectivamente 


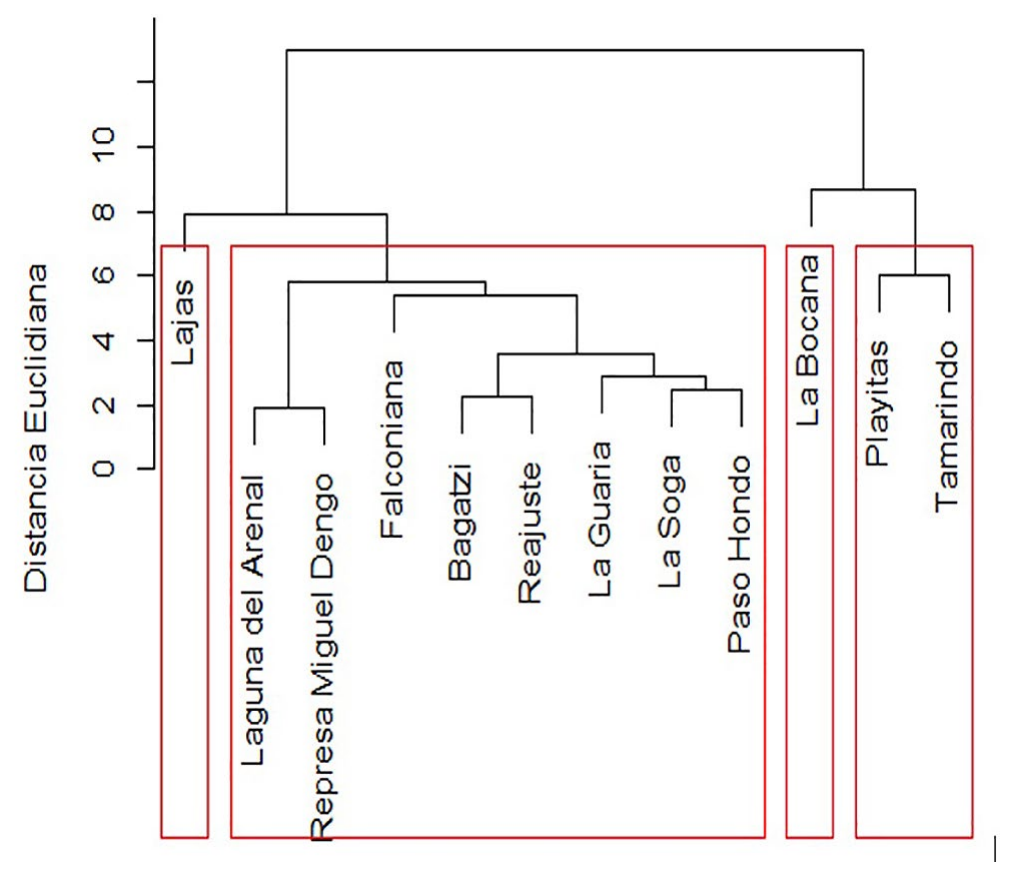

Figura 5. Dendrograma de los puntos de muestreo

\subsection{Análisis de Conglomerados o Clúster (AC)}

Se realiza un análisis de conglomerados jerárquicos, cuyo dendrograma se percibe en la Fig. 5, donde las variables se separan en cuatro categorías:

- Laguna Bocana: se mantiene independiente, lo que concuerda con los gráficos 4.b y 4.d. Lo anterior se refleja en los promedios de porcentaje de saturación de oxígeno y oxígeno disuelto que son los mínimos reportados de 7,48\% y $0,84 \mathrm{mg} / \mathrm{L}$, respectivamente;

- $\quad$ Lajas: está diferenciado, coincidiendo con los resultados de las Fig. 4.b y 4.f. Se posiciona en la sección final del Canal Sur (Fig. 1) y se asocia con los promedios máximos de las dos variables que conforman el CP2: SAAM (1,6 mg/L) y nitrógeno total (4,44 mg/L);

- $\quad$ Playitas y Tamarindo: se asemejan entre sí formando un conglomerado, son los dos sitios que muestran los valores máximos más altos de coliformes totales. Playitas reporta 540000 NMP/100 mL de CF y en Tamarindo es 2400000 NMP/100 mL de CT;

- $\quad$ En el grupo más general están Laguna del Arenal y la represa MPDB, que corresponden a las fuentes de agua del DRAT y separándose de los seis puntos restantes: Falconiana, Bagatzí, Reajuste, La Guaria, La Soga y Paso Hondo.

\subsection{Análisis de Discriminante (AD)}

El análisis de conglomerados (AC) es una herramienta que permite agrupar los lugares de muestreo, como base para definir los grupos a priori (original) que alimentan el análisis de discriminante 
(AD). Se obtiene que el Lambda de Wilks es de 0,39; que es moderamente bajo indicando un menor solapamiento entre los grupos. Además, se reporta un valor de $\mathrm{p}<0,0001$; de tal modo que existen diferencias significativas entre los centroides de los clústers.

Para la categorización de los cuatro grupos se debe trabajar con tres funciones discriminantes, cuyos coeficientes se plantean en la Tabla IV. Los autovalores para la función discriminante canónica explican para la primera el 66,18\%, para la segunda el 26,99\% y para la tercera el 6,83\% de variación entre los grupos.

En la función discriminante canónica propuesta en la Tabla IV con las 22 variables, los coeficientes responden a las distribuciones de cada variable. En este estudio las variables tienen varianzas muy distintas; por lo que es más apropiada la aplicación de la función discriminante estandarizada por las covarianzas comunes (Tabla IV). Además, se aprecia que los sólidos totales y los disueltos son los parámetros que más inciden en la separación de grupos, mostrando concordancia con los resultados del primer componente principal del ACP.

TABLA IV

COEFICIENTES DE LAS FUNCIONES DISCRIMINANTES CANÓNICAS Y DISCRIMINANTE ESTANDARIZADA POR LAS COVARIANZAS COMUNES

\begin{tabular}{|c|c|c|c|c|c|c|}
\hline \multirow[t]{2}{*}{ Parámetros } & \multicolumn{3}{|c|}{ Discriminante Canónico } & \multicolumn{3}{|c|}{ Discriminante Estandarizado } \\
\hline & 1 & 2 & 3 & 1 & 2 & 3 \\
\hline Calcio $\left(\mathrm{Ca}^{2+}\right)$ & $-4,4 \mathrm{E}-03$ & 0,02 & $-0,04$ & $-0,05$ & 0,42 & $-0,36$ \\
\hline Coliformes fecales (CF) & $-5,1 \mathrm{E}-07$ & $-2,8 \mathrm{E}-06$ & $-3,3 \mathrm{E}-06$ & $-0,04$ & $-0,22$ & $-0,24$ \\
\hline Coliformes totales (CT) & $4,3 \mathrm{E}-07$ & $-1,6 \mathrm{E}-06$ & $1,4 \mathrm{E}-07$ & 0,09 & $-0,31$ & $3,9 \mathrm{E}-03$ \\
\hline Conductividad eléctrica (CE) & $1,3 \mathrm{E}-03$ & $7,0 \mathrm{E}-04$ & $2,8 \mathrm{E}-04$ & 0,21 & 0,02 & 0,03 \\
\hline Demanda bioquímica de oxígeno (DBO) & $-0,03$ & $-0,06$ & 0,03 & $-0,10$ & $-0,23$ & 0,06 \\
\hline Demanda química de oxígeno (DQO) & 0,01 & $1,8 \mathrm{E}-03$ & $-1,4 \mathrm{E}-03$ & 0,26 & 0,06 & $-0,08$ \\
\hline Dureza total (DT) & $-8,0 \mathrm{E}-04$ & $9,5 \mathrm{E}-03$ & $-3,3 \mathrm{E}-03$ & $-0,03$ & 0,45 & $-0,05$ \\
\hline Fósforo total (F) & $-0,15$ & 0,07 & $-1,77$ & $-0,04$ & 0,01 & $-0,48$ \\
\hline Grasas y aceites (GyA) & $-0,01$ & $-0,05$ & $-0,06$ & $-0,03$ & $-0,28$ & $-0,29$ \\
\hline Magnesio $\left(\mathrm{Mg}^{2+}\right)$ & 0,03 & $-0,08$ & 0,25 & 0,12 & $-0,27$ & 0,87 \\
\hline Nitrógeno total $(\mathrm{N})$ & $-0,11$ & 0,39 & $-0,44$ & $-0,07$ & 0,23 & $-0,30$ \\
\hline Oxígeno disuelto (OD) & $-0,29$ & 0,15 & $-0,01$ & $-0,54$ & 0,17 & $-0,09$ \\
\hline Potencial de hidrógeno $(\mathrm{pH})$ & 0,16 & $-0,29$ & $-0,63$ & 0,07 & $-0,04$ & $-0,23$ \\
\hline Potasio $\left(\mathrm{K}^{+}\right)$ & $-0,18$ & $-0,37$ & $-0,04$ & $-0,43$ & $-0,88$ & $-0,01$ \\
\hline $\begin{array}{l}\text { Sustancias activas al azul de metileno } \\
\text { (SAAM) }\end{array}$ & 2,70 & 0,76 & 2,53 & 0,38 & 0,15 & 0,36 \\
\hline Saturación de oxígeno (SO) & $7,2 \mathrm{E}-04$ & $2,9 \mathrm{E}-04$ & $2,4 \mathrm{E}-04$ & 0,02 & 0,06 & 0,03 \\
\hline Sólidos disueltos (SD) & $2,8 \mathrm{E}-03$ & 0,02 & $-0,01$ & 0,26 & 1,26 & $-1,55$ \\
\hline Sólidos sedimentables (SS) & $-0,11$ & 0,22 & 1,98 & $-0,03$ & 0,13 & 0,61 \\
\hline Sólidos suspendidos totales (SST) & $-0,01$ & 0,02 & $-0,01$ & $-0,57$ & 0,67 & $-0,59$ \\
\hline Sólidos totales (ST) & 0,01 & $-0,01$ & 0,01 & 0,94 & $-1,14$ & 1,21 \\
\hline Temperatura $(\mathrm{T})$ & 0,02 & $-0,02$ & $-0,17$ & 0,04 & $-0,08$ & $-0,38$ \\
\hline
\end{tabular}


TABLA V

MATRIZ DE CLASIFICACIÓN DEL ANÁLISIS DE DISCRIMINANTE

\begin{tabular}{lccccc}
\hline & \% Correcto & La Bocana & Lajas & $\begin{array}{c}\text { Playitas y } \\
\text { Tamarindo }\end{array}$ & General \\
\hline Original & 100,00 & 5 & 0 & 0 & 0 \\
La Bocana & 100,00 & 0 & 4 & 0 & 0 \\
Lajas & 91,67 & 1 & 0 & 11 & 0 \\
Playitas y Tamarindo & 83,18 & 1 & 7 & 10 & 89 \\
General & 85,16 & & & & 2 \\
Total & & & & & 2 \\
Validación Cruzada & 60,00 & 3 & 0 & 0 & 4 \\
La Bocana & 40,00 & 1 & 2 & 7 & \\
Lajas & 53,85 & 1 & 1 & 14 & \\
Playitas y Tamarindo & 77,14 & 2 & 8 & & \\
General & 72,66 & & & & \\
Total & & & & & \\
\hline
\end{tabular}

La Tabla V muestra que los datos originales son correctamente clasificados en un $85,16 \%$, donde la distribución de errores no es uniforme, presentándose el mayor número de casos mal categorizados en el grupo general; que corresponde al de mayor tamaño; que podría mejorarse con una variación del punto de corte a partir de un análisis de probabilidades. Mientras que en el método de validación cruzada se observa que la tasa de clasificación correcta disminuye en todos los grupos, para alcanzar un total de 72,66 \% . Las funciones de clasificación para futuras muestras se estimarían con una evaluación más realista del modelo.

El agrupamiento de los puntos de muestreo con base en los resultados del AC y del AD permite asociar los parámetros que presentan mayor variabilidad en sitios de descarga de aguas específicos; facilitando la identificación de problemáticas correspondientes al uso del recurso hídrico de manera espacial.

\section{CONCLUSIONES}

El presente estudio evalúo el conjunto de 12 ubicaciones en el DRAT, donde se realiza el control de calidad de agua abarcando un período de una década, con el uso de técnicas estadísticas utilizadas como análisis de componentes principales, análisis de conglomerados y análisis de discriminante. Estas técnicas permitieron ir más allá de los resultados de muestreos puntuales semestrales, obteniendo resultados vinculantes entre los métodos aplicados, así resalta las problemáticas asociadas con el manejo de aguas de vertido para sistemas de irrigación como los son la salinidad o la contaminación fecal.

A partir de la investigación se logra relacionar puntos espaciales del DRAT con las variables microbiológicas, físicas y/o químicas que producían mayor impacto en los mismos; por lo que se 
considera un recurso inicial de control de calidad que permite generar programas para el mejoramiento y conservación del recurso hídrico de la zona.

Se recomienda extender la investigación para evaluar el comportamiento hidrológico e hidrogeoquímico que permita explicar el transporte de contaminantes en aguas superficiales, así como realizar un tratamiento de los datos para la época seca y lluviosa cuando se cuente con un registro más extenso de datos, principalmente en los sitios donde no fue posible la determinación de la calidad del agua por ausencia de caudal.

\section{AGRADECIMIENTOS}

Un agradecimiento especial al Ing. Reiner Sibaja Matarrita por su colaboración con la información brindada por el Proyecto N340-B5-507 “Optimización del Recurso Hídrico en el Sistema de Riego Arenal Tempisque”, inscrito en la Vicerrectoría de Investigación de la Universidad de Costa Rica.

Gracias a la cooperación dada por los personeros del DRAT, que facilitaron la base de datos para este estudio, así como su participación y retroalimentación para la interpretación de los resultados.

Además, un agradecimiento a todos los revisores del artículo, pues sus recomendaciones y comentarios, han sido muy valiosos y un gran aporte para la calidad del presente trabajo.

\section{REFERENCIAS}

[1] FAO, "Buenas perspectivas para los sistemas de riego con energía solar." http://www.fao.org/news/ story/es/item/1116521/icode/ (accessed Sep. 28, 2020).

[2] I. García-Garizábal and J. Causapé, "Influence of irrigation water management on the quantity and quality of irrigation return flows," J. Hydrol., vol.385, no. 1-4, pp. 36-43, May 2010, doi: 10.1016/j. jhydrol.2010.02.002.

[3] Á. García, “Criterios modernos para evaluación de la calidad del agua para riego,” 2012. Accessed: Jan. 12, 2021. [Online]. Available: http://ipni.net/publication/ia-lahp.nsf/0/6E4999FFE5F6B8F005257A920059B3B6/\$FILE/Art 5.pdf.

[4] P. S. Minhas, T. B. Ramos, A. Ben-Gal, and L. S. Pereira, "Coping with salinity in irrigated agriculture: Crop evapotranspiration and water management issues," Agricultural Water Management, vol. 227. Elsevier B.V., p. 105832, Jan. 20, 2020, doi: 10.1016/j.agwat.2019.105832.

[5] O. Heredia, "El agua de Riego: Criterios de Interpretación. Efectos sobre el suelo y la producción," in Agua subterránea. Exploración y utilización agropecuaria, Argentina: EFA-Editorial Facultad de Agronomía, Universidad de Buenos Aires, 2006, pp. 75-99.

[6] D. L. Suarez, J. D. Wood, and S. M. Lesch, "Effect of SAR on water infiltration under a sequential rain-irrigation management system," Agric. Water Manag., vol. 86, no. 1-2, pp. 150-164, Nov. 2006, doi: 10.1016/j.agwat.2006.07.010.

[7] M. A. Neira Gutiérrez, "Dureza en aguas de consumo humano y uso industrial, impactos y medidas de mitigación. Estudio de caso: Chile," Tesis de Pregrado de Ingeniería Civil, Universidad de Chile, Chile, 2006. 
[8] J. Causapé, D. Quílez, and R. Aragüés, "Assessment of irrigation and environmental quality at the hydrological basin level: II. Salt and nitrate loads in irrigation return flows," Agric. Water Manag., vol. 70, no. 3, pp. 211-228, Dec. 2004, doi: 10.1016/j.agwat.2004.06.006.

[9] Y. Pachepsky, D. R. Shelton, J. E. T. McLain, J. Patel, and R. E. Mandrell, "Irrigation Waters as a Source of Pathogenic Microorganisms in Produce: A Review," in Advances in Agronomy, vol. 113, Academic Press, 2011, pp. 75-141.

[10] G. Kisluk and S. Yaron, "Presence and persistence of salmonella enterica serotype typhimurium in the phyllosphere and rhizosphere of spray-irrigated parsley," Appl. Environ. Microbiol., vol. 78, no. 11, pp. 4030-4036, Jun. 2012, doi: 10.1128/AEM.00087-12.

[11] O. O. Alegbeleye, I. Singleton, and A. S. Sant'Ana, "Sources and contamination routes of microbial pathogens to fresh produce during field cultivation: A review," Food Microbiology, vol. 73. Academic Press, pp. 177-208, Aug. 01, 2018, doi: 10.1016/j.fm.2018.01.003.

[12] M. Uyttendaele et al., "Microbial Hazards in Irrigation Water: Standards, Norms, and Testing to Manage Use of Water in Fresh Produce Primary Production," Compr. Rev. Food Sci. Food Saf., vol. 14, no. 4, pp. 336-356, Jul. 2015, doi: 10.1111/1541-4337.12133.

[13] C. Leifert, K. Ball, N. Volakakis, and J. M. Cooper, "Control of enteric pathogens in ready-to-eat vegetable crops in organic and 'low input' production systems: a HACCP-based approach," J. Appl. Microbiol., vol. 105, no. 4, pp. 931-950, Oct. 2008, doi: 10.1111/j.1365-2672.2008.03794.x.

[14] K. A. Hamilton, W. Ahmed, E. Rauh, C. Rock, J. McLain, and R. L. Muenich, “Comparing microbial risks from multiple sustainable waste streams applied for agricultural use: Biosolids, manure, and diverted urine," Current Opinion in Environmental Science and Health, vol. 14. Elsevier B.V., pp. 37-50, Apr. 01, 2020, doi: 10.1016/j.coesh.2020.01.003.

[15] P. K. Jjemba, L. A. Weinrich, W. Cheng, E. Giraldo, and M. W. LeChevallier, "Regrowth of potential opportunistic pathogens and algae in reclaimed-water distribution systems," Appl. Environ. Microbiol., vol. 76, no. 13, pp. 4169-4178, Jul. 2010, doi: 10.1128/AEM.03147-09.

[16] S. Liu et al., "Characterisation of spatial variability in water quality in the Great Barrier Reef catchments using multivariate statistical analysis," Mar. Pollut. Bull., vol. 137, pp. 137-151, Dec. 2018, doi: 10.1016/j.marpolbul.2018.10.019.

[17] H. S. Jahin, A. S. Abuzaid, and A. D. Abdellatif, "Using multivariate analysis to develop irrigation water quality index for surface water in Kafr El-Sheikh Governorate, Egypt," Environ. Technol. Innov., vol. 17, p. 100532, Feb. 2020, doi: 10.1016/j.eti.2019.100532.

[18] N. Magyar, I. G. Hatvani, I. K. Székely, A. Herzig, M. Dinka, and J. Kovács, “Application of multivariate statistical methods in determining spatial changes in water quality in the Austrian part of Neusiedler See," Ecol. Eng., vol. 55, pp. 82-92, Jun. 2013, doi: 10.1016/j.ecoleng.2013.02.005.

[19] B. Shah et al., "Reckoning of water quality for irrigation and drinking purposes in the konkan geothermal provinces, Maharashtra, India," Groundw. Sustain. Dev., vol. 9, p. 100247, Oct. 2019, doi: 10.1016/j.gsd.2019.100247.

[20] M. Hajigholizadeh and A. M. Melesse, "Assortment and spatiotemporal analysis of surface water quality using cluster and discriminant analyses," Catena, vol. 151, pp. 247-258, Apr. 2017, doi: 10.1016/j.catena.2016.12.018.

[21] M. S. Samsudin, A. Azid, S. I. Khalit, M. S. A. Sani, and F. Lananan, "Comparison of prediction model using spatial discriminant analysis for marine water quality index in mangrove estuarine zones," Mar. Pollut. Bull., vol. 141, pp. 472-481, Apr. 2019, doi: 10.1016/j.marpolbul.2019.02.045. 
[22] U. C. R. Escuela de Ingeniería de Biosistemas, "Sistema de Apoyo para la Gestión Inteligente del Recurso Hídrico (SAGIRH).” https://www.sagirh.ucr.ac.cr/drat/ (accessed Sep. 28, 2020).

[23] IMN, “Estaciones Meteorológicas Automáticas de Costa Rica.” https://www.imn.ac.cr/estaciones-automaticas (accessed Feb. 16, 2021).

[24] E. J. Acuña, "Modelo de operación del sistema de canales del Distrito de Riego Arenal-Tempisque," Tesis de Licenciatura de Escuela de Ingeniería Civil, Universidad de Costa Rica, Costa Rica, 2016.

[25] SENARA, "Dirección de Riego Arenal Tempisque.” http://www.senara.or.cr/drat/index.aspx (accessed Jan. 06, 2021).

[26] Ministerio de Ambiente y Energía, "Reglamento de vertido y reúso de aguas residuales," San José, Costa Rica, 2007. Accessed: Oct. 03, 2020. [Online]. Available: http://www.digeca.go.cr/sites/default/ files/reglamento_vertido_reuso_aguas_residuales_0.pdf.

[27] APHA, AWWA, and WEF, Standard Methods for the Examination of Water and Wastewater, 22 th Edition. Washington, 2012.

[28] S. de la Fuente Fernández, "Análisis componentes principales (ACP)," Facultad de Ciencias Económicas y Empresariales de la Universidad Autónoma de Madrid, España, 2011.

[29] J. A. Di Rienzo, F. Casanoves, M. G. Balzarini, L. González, E. Tablada, and C. W. Robledo, "Infostat - Software estadístico.” Universidad Nacional de Córdoba, Argentina, 2008, Accessed: Jan. 13, 2021. [Online]. Available: https://www.infostat.com.ar/.

[30] S. Lê, J. Josse, and F. Husson, "FactoMineR: An R package for multivariate analysis," J. Stat. Softw., vol. 25, no. 1, pp. 1-18, Mar. 2008, doi: 10.18637/jss.v025.i01.

[31] S. Dray and A.-B. Dufour, "The ade4 Package: Implementing the duality diagram for e cologists," $J$. Stat. Softw., vol. 22, no. 4, pp. 1-20, 2007, doi: 10.18637/jss.v022.i04.

[32] W. N. Venables and B. Ripley, "Modern Applied Statistics with S, 4th ed," 2002. http://www.stats. ox.ac.uk/pub/MASS4/ (accessed Jan. 12, 2021).

[33] World Health Organization, "Hardness in drinking," Suiza, 2011. Accessed: Oct. 03, 2020. [Online]. Available: https://www.who.int/water_sanitation_health/dwq/chemicals/hardness.pdf.

[34] A. Kulkarni, "Water quality retrieval from landsat TM imagery," in Procedia Computer Science, Jan. 2011, vol. 6, pp. 475-480, doi: 10.1016/j.procs.2011.08.088.

[35] J. J. Alvarado and J. F. Aguilar, "Batimetría, salinidad, temperatura y oxígeno disuelto en aguas del Parque Nacional Marino Ballena, Pacífico, Costa Rica,” Rev. Biol. Trop., vol. 57 (1), pp. 19-29, 2009, Accessed: Oct. 03, 2020. [Online]. Available: http://repositoriosiidca.csuca.org/Record/ RepoKERWA25756.

[36] US EPA, "Indicators: Dissolved Oxygen," Aug. 16, 2016. https://www.epa.gov/national-aquatic-resource-surveys/indicators-dissolved-oxygen (accessed Jan. 13, 2021).

[37] A. C. Rencher, "Frontmatter," in Methods of Multivariate Analysis, New York, NY, USA: John Wiley \& Sons, Inc., 2003, pp. i-xxii.

[38] Convenio Ramsar, "Sitio Ramsar: Parque Nacional Palo Verde, Costa Rica," San José, 1998. Accessed: Oct. 03, 2020. [Online]. Available: https://www.ramsar.org/sites/default/files/documents/library/ ram39s_palo_verde_cont._informe_combined.pdf. 